\title{
Analysis of Leaf Trait and Differentially Expressed Genes of Leaf Development in Eucommia ulmoides Oliver after Ultraviolet-B (UV-B) Radiation
}

\section{Li Dewen}

Northeast Forestry University

\section{Liu Ying}

Northeast Forestry University https://orcid.org/0000-0002-2303-3791

\section{Du Dandan}

Northeast Forestry University

\section{Zu Yuangang}

Northeast Forestry University

\section{Tang Zhonghua}

Northeast Forestry University

Zhao Yusen ( $\nabla$ zhaoysnefu@126.com )

Northeast Forestry University

\section{Original Article}

Keywords: Leaf development, Transcriptome, Eucommia ulmoides Oliver, UV-B

Posted Date: October 29th, 2020

DOI: https://doi.org/10.21203/rs.3.rs-97641/v1

License: (9) (i) This work is licensed under a Creative Commons Attribution 4.0 International License. Read Full License 


\section{Abstract}

\section{Background}

Eucommia ulmoides Oliver (EUO) has a great medicinal value in China. The using leaf model of Eucommia planting (ULMEP) is a promising approach to resolve the shortage of EUO resources. We focused on leaf responses and differentially expressed genes (DEGs) for transcriptome profiling after ultraviolet-B radiation (UV-B) in the east-north China.

\section{Results}

The leaf traits were significantly affected after UV-B treatment, and the leaf area, leaf thickness and total leaf fresh/dry weight significantly increased $(p<0.05)$. Anatomical results showed that cells from the leaf epidermal and palisade tissue were larger than those of control. Two transcriptome databases were assembled and integrated, the transcriptome databases comparative analysis revealed 1,826 upregulated and 1,872 down-regulated genes. Among them, the up-regulated unigenes were mainly participated in Signal transduction mechanisms, and Cell wall/membrane biogenesis by the KOG database, and up-regulated genes were analyzed in plant hormone signal transduction and phenylpropanoid metabolic pathways by KEGG pathway. Furthermore, randomly 10 candidate unigenes expression were examined using RT-qPCR, the two libraries exhibited differential expression and were same as those obtained by sequencing.

\section{Conclusions}

This study was helpful to clarify the cause of leaf enlargement after UV-B treatment, the differentially expressed genes (DEGs) from plant auxin and phenylpropanoid biosynthesis signal pathways were focused on after UV-B treatment. This study provided a valuable reference for the ULMEP in the eastnorth China.

\section{Background}

Eucommia ulmoides Oliver (EUO) is used as a component of traditional medicine. The bark and leaves can be used to identify the material in Chinese traditional medicine. The traditional forest model of Eucommia planting (AFMEP) is kinds of tall trees (height of 10-40 m) [Wang et al. 2018]. The traditional way to utilize the valuable parts (such as the bark) of EUO is to cut down all the tree, thus damaging the environment and resource. To change these shortcomings, Su's study group has achieved using leaf model of Eucommia planting (ULMEP) to generate trees with a 2-3 m height, and for the collection of a great number of branches and leaves [Zhu et al.2017]. The ULMEP can produce a large amount of valuable parts with leaves and branches. Thus, researchers have become increasingly interested in the ULMEP. In addition, EUO is widely distributed in the middle and north subtropical and temperate regions in China, but the development of ULMEP in the northeast (Harbin) is not much research. 
Ultraviolet-B (UV-B) radiation (280-315 nm) is undoubtedly only a small fraction of the solar radiation, the most important environmental factor affecting leaf developmental changes [Dotto et al.2017]. Currently, some plant growth cannot be limited after UV-B radiation [Rinella and James.2017]. plants might be to perceive this radiation as a signal without stress treatment [Hectors et al.2007]. Because UV-B radiation can induce the rapid expression of a variety of genes, for example, including those involved in the synthesis of phenylpropanoids [Wargent et al.2009; Zhu et al.2016], which could influence the developmental processes throughout their lifetime. Therefore, the purpose of this study is to try to change the development of ULMEP after UV-B radiation in the northeast of China.

RNA-Seq has been a popular tool for large-scale sequencing without the requirement for a reference genome [Grabherr et al.2011]. To date, the transcriptome of the EUO flower bud development, and transcriptome identification of genes related to salt stress in EUO have been sequenced [Wang et al. 2018; Liu et al.2016]. In our study, we identified differentially expressed genes (DEGs) by applied Illumina sequencing technology in the changes of leaf development after UV-B radiation.

\section{Materials And Methods}

Plant material and experimental design

Our experiment was done outside for one growing season (from May 15, 2016 to June 15, 2017) at the North-East Forest University, Heilongjiang Province, China ( $45.75^{\circ} \mathrm{N}, 126.63^{\circ} \mathrm{E}, 1,826 \mathrm{~m}$ a.s.I.). Uniform five-year-old Eucommia ulmoides Oliver (EUO) seedlings were transplanted into plastic pots (35-cm depth and 25-cm diameter) and a seedling per pot. The substrate used for growing the seedlings was peat: vermiculite: perlite $(1: 1: 1, \mathrm{v} / \mathrm{v} / \mathrm{v})$ without fertilization at $26^{\circ} \mathrm{C}$ under natural light. The experiment set up two treatments: (1) natural solar radiation (control, C) and (2) ultraviolet-B (UV-B) radiation. The UV-B treatment provided by fluorescent tube lamps (Beijing Electronic Resource Institute, Beijing, China) on the metal frames. The distance from the lamps to the top of the plant apex was $80 \mathrm{~cm}$ and was remained unchanged throughout the experiment. Vertical polyester curtains were used to prevent UV-B radiation from radiating the control seedlings. The supplemental UV-B dose was $1.30 \mathrm{~kJ} \cdot \mathrm{m}^{-2} \cdot \mathrm{d}^{-1}\left(3.3 \mathrm{mmol} \cdot \mathrm{m}^{-2} \cdot \mathrm{d}^{-}\right.$ $\left.{ }^{1}\right)$. The seedlings were irradiated for $8 \mathrm{~h}$ daily throughout a week, centered on the solar noon, and then seedlings of all pots from the control and treatment were carefully planted according to leaf model of Eucommia planting (ULMEP). After one year of ecological restoration, the leaf materials were collected from July 2017. The leaf samples were frozen in liquid nitrogen for RNA extraction, and the other samples were deposited at $4^{\circ} \mathrm{C}$ for microstructure observation.

\section{Leaf growth parameters}

Six randomly selected seedlings from each treatment replicate were harvested in July. The leaf area was determined by Portable Laser Area Meter (Cl-203, CID Inc., USA). Fine specific leaf area (SLA, leaf area per unit leaf mass) was calculated. Leaf thickness was measured by a photomicroscope fitted with a micrometer, employing 25 semithin transverse sections cut from leaf material. All statistical analyses 
were performed using the Software Statistical Package for the Social Science (SPSS) version

\section{Determination of leaf anatomy}

Leaf samples for trans-section examinations were cut into pieces and fixed in FAA solution. Fixed samples were dehydrated by a graded ethanol series, and immersed by a graded ethanol series, then embedded in paraffin for sectioning; transverse sections were made on a Leica 2016 rotary microtome (Leica Inc., Bensheim, Germany), mounted on glass slides, then treated with a safranin and fast-green staining procedure, and dehydrated through an ethanolic series. Sections were observed by a Nikon (TE2000-U) electron microscope equipped with a $20 \times 0.45$ objective lens, and images were obtained.

\section{Illumina sequencing for transcriptome analysis}

Total RNA from the two samples from the leaves of control and UV-B treatment were extracted using TRIzol (Sangon, Shanghai, China) following the manufacturer's instructions and then treated with RNasefree DNase I. Preparation of the cDNA was described in detail in Liu's method [Liu et al.2016]. The equal amounts of total RNA were pooled from three biological replicates. The two libraries were used for comparative transcriptome sequencing analysis. Two libraries were sequenced at the Beijing ORI-gene Institute (ORI, Beijing, China). All raw transcriptome data were stored in the GenBank Short Read Archive.

\section{De novo assembly and functional analysis of Illumina sequencing}

Raw reads from the sequencing platform were generated by base calling, referring to the Conesea's method [Conesa et al.2005]. These contigs were further processed by sequence clustering software TGICL to form longer sequences, which were defined as unigenes. The generated unigenes were used for BLASTX alignment $(E<0.00001)$ and annotation against protein databases, including nonredundant (NR), SwissProt, Clusters of orthologous groups for eukaryotic complete genomes (KOG), and Kyoto Encyclopedia of Genes and Genomes (KEGG) protein databases. With NR annotation, the Gene Ontology (GO) annotation of unigenes were performed by using the Blast2GO program [Conesa et al.2005], GO functional classification for all unigenes were obtained through WEGO software [Ye et al.2006].

\section{Identification of differentially expressed genes (DEGs)}

To compare the differences gene expression in leaves at control and UV-B radiation treatments, the read density was calculated by RPKM method (reads per kb per million reads). The FDR (false discovery rate) was used to determine the threshold P-value in multiple tests. According to Zhang's [Zhang et al.2016] approach, we used an FDR $<0.01, P \leq 0.05$ and an absolute value of the $\log _{2}$ ratio $>1$ as the threshold to determine significant differences in gene expression.

\section{The validation of quantitative real-time PCR}

Total RNAs were extracted as described above. Each total RNA (2 mg) were subjected to reverse transcriptase reactions using the RevertAid H Minus First-Strand cDNA Synthesis Kit (MBI Fermentas, Canada) according to the company instructions. The specific primers sequences are listed in table 1 . The 
constitutive expressed gene of ribosomal 40S protein S9 (rps9, AJ749993.1), was used as the internal control. The first-strand cDNA was used in RT-qPCR, each reaction contained a mixture of $1.5 \mu$ l of diluted cDNA (diluted 20 times with nuclease-free water) aliquots, $1.5 \mu \mathrm{l}$ mixed primers, $10 \mu \mathrm{l}$ mixture of BeyoFast $^{\mathrm{TM}}$ SYBR Green qPCR (Beyotime, Nanjing, China) and $6.0 \mu \mathrm{l}$ of nuclease-free water. RT-qPCR amplification were carried out in 96-well plates on a LightCycler ${ }^{\circledR} 480$ II System (Roche, Switzerland Roche Diagnostics, Indianapolis, IN, USA). The qRT-PCR results were obtained from three technical repeats for each gene and sample.

Table 1 Primer sequences of the reference gene and candidate unigenes for qRT-PCR. 


\begin{tabular}{|c|c|}
\hline Primer & Sequences $\left[5^{\prime}-3^{\prime}\right]$ \\
\hline \multirow[t]{2}{*}{ rps9 } & GGT TGT CAA TGT TCC TTC CTT C \\
\hline & ТСТ ТСА ТСС ТСТ ТСА ТСТ ССА ТО \\
\hline \multirow[t]{2}{*}{ TR11546 } & GGA TGC AGA ATC GGG AAG GA \\
\hline & ATC AAA TGC CGG ATT CGC CT \\
\hline \multirow[t]{2}{*}{ TR11244 } & TGT CAC TGG AAG AGA CGG GA \\
\hline & AAT CGA TCC CAC CTC GTG TG \\
\hline \multirow[t]{2}{*}{ TR11203 } & TGG CGT ATA GTG ACA TGG GC \\
\hline & CAA CGT AAG AGG GAA GGC GT \\
\hline \multirow[t]{2}{*}{ TR10148 } & GTA CCT GGC CCG ATC ATA GC \\
\hline & CAG AAG CAA TGG GTG GGG AT \\
\hline \multirow[t]{2}{*}{ TR11070 } & TTC CCC AAG GTA CAT CCC CA \\
\hline & GAC TGA CAC CTT GAT CGG CA \\
\hline \multirow[t]{2}{*}{ TR14945 } & ACG TTT ATG GGT TGT CGG CT \\
\hline & ATC GCC CAT TTT CTC ACC GT \\
\hline \multirow[t]{2}{*}{ TR10048 } & ACA ATT TCG CTC CCG TCC AT \\
\hline & TGG TTC GAG GTT GTC TCT GC \\
\hline \multirow[t]{2}{*}{ TR19418 } & CTC GGC TGA CCC AGA AAC AT \\
\hline & CCA TAC CGC CTC ACC AAA CT \\
\hline \multirow[t]{2}{*}{ TR8189 } & TCG CCA TTA GGG CTT GAT CC \\
\hline & CAC ACA CAA TCG GAT TCG CC \\
\hline \multirow[t]{2}{*}{ TR15898 } & TGC GTT TTT AAC CCA CTG CG \\
\hline & AGA CGA ATT GCC ACC CTG AG \\
\hline
\end{tabular}

\section{Results}

\section{Leaf growth parameters}

The leaf growth traits were significantly different from control after UV-B treatment (Table 2). The leaf area was increased by 1.98 times, the leaves number, leaf width, leaf length and whole-plant fresh weight (FW) increased after UV-B radiation, and were $132.0 \pm 6.6,9.24 \pm 0.46 \mathrm{~cm}, 17.32 \pm 0.87 \mathrm{~cm}$, and $247 \pm$ 
$12.36 \mathrm{~g}$, respectively. The results from table 1 showed that leaf thickness and biomass were significantly higher than that of the control after UV treatment.

Table 2 The effects of UV-B treatment on the growth parameters of EUO leaves

\begin{tabular}{|c|c|c|}
\hline Leaf traits & Control & UV-B \\
\hline Number of leaves & $139.00 \pm 6.95^{a}$ & $132.00 \pm 6.6^{a}$ \\
\hline Leaf width $(\mathrm{cm})$ & $6.62 \pm 0.33^{a}$ & $9.24 \pm 0.46^{a}$ \\
\hline Leaf length (cm) & $12.59 \pm 0.63^{b}$ & $17.32 \pm 0.87^{a}$ \\
\hline Leaf area $\left(\mathrm{cm}^{2}\right)$ & $61 \pm 3.05^{b}$ & $121 \pm 6.05^{a}$ \\
\hline Leaf thickness (mm) & $0.23 \pm 0.012^{b}$ & $0.31 \pm 0.016^{a}$ \\
\hline Leaf fresh weight (g) & $163.77 \pm 8.19^{b}$ & $247.12 \pm 12.36^{a}$ \\
\hline Leaf dry weight (g) & $57.39 \pm 2.87^{b}$ & $92.84 \pm 4.64^{\mathrm{a}}$ \\
\hline
\end{tabular}

Values are means \pm SD from 30 developing leaves.

Different lower-case letters show significant differences according to Duncan's multiple test $(P<0.05)$. Leaf anatomy analysis

Anatomical observation of leaf cross-sections indicated that the epidermal cells were larger than the control (Fig. 1). Compared with control, the palisade tissue became long and loose, and the spaces between cells increased, the cells of the spongy tissue deform and become dispersed (Fig. 2). The palisade tissue cells of the leaf after UV-B treatment were 2.5 times larger than that of the control. These results indicate that the anatomy of the cells in the leaves changed significantly after UV-B treatment.

\section{De novo assembly and sequence annotation}

The clean reads were assembled into 62,287 transcripts and 42,333 unigenes using Trinity, among a total length of 70,449,627 bp and 41,056,860 bp, a mean length of 1,131 bp and $969 \mathrm{bp}$, and an N50 of 1,762 bp and 1,582 bp, respectively (Fig 2a). The quality of the whole sequencing was high and thus adequate for follow-up analyses. The $200-1000$ bp transcripts accounted for $62 \%$ of the total transcripts; $22 \%$ $(13,533)$ of the transcripts ranged from 1,100 to $1,900 \mathrm{bp}$; and $10,045(16 \%)$ of the transcripts were longer than $2,000 \mathrm{bp}$. Most $(29,473)$ of the unigenes ranged from 201 to $1,000 \mathrm{bp}$, accounting for $70 \%$ of the total unigenes; $18 \%(7,700)$ of the unigenes ranged from 1,100 to $1,900 \mathrm{bp}$; and $5,159(12 \%)$ of the unigenes were longer than 2,000 bp (Fig. 2a). The differentially expressed genes (DEGs) were screened, and an MA map was generated to display the level of expression of all genes between groups (FPKM) and the distribution of DEGs (Fig. 2b). Differential gene cluster analysis showed that there were significant differences in gene expression patterns between the control and the UV-B treatment (Fig. 2c). 


\subsection{Sequence annotation and classification}

After aligning the unigene sequences to protein databases, 42,333 unigenes were annotated. The percentage and numbers of unigenes annotated within the SwissProt, TrEMBL, nonredundant (NR), Pfam, Clusters of orthologous groups for eukaryotic complete genomes (KOG), Gene Ontology (GO) and Kyoto Encyclopedia of Genes and Genomes (KEGG) Orthology (KO) databases were 14,988 (35.40\%), 24,272 (57.30\%), 24,500 (57.90\%), 19,708 (46.60\%), 19,523 (46.10\%), 19,282 (45.5\%) and 6,790 (16\%), respectively (Table 2 ).

Table 2 The number of unigenes annotated to different databases

\begin{tabular}{|llllllll|}
\hline Unigene & SwissProt & TrEMBL & NR & Pfam & KOG & GO & KO \\
\hline 42333 & 14988 & 24272 & 24500 & 19708 & 19523 & 19282 & 6790 \\
\hline $100 \%$ & $35.40 \%$ & $57.30 \%$ & $57.90 \%$ & $46.60 \%$ & $46.10 \%$ & $45.50 \%$ & $16.00 \%$ \\
\hline
\end{tabular}

\section{Functional classification of differentially expressed genes}

The Fig 3a showed that the up-regulated genes involved in the translation, ribosomal structure and biogenesis[J] and Transcription $[\mathrm{K}]$ among the 19 categories in the information storage and processing; in the cellular processes and signaling, the up-regulated genes involved in posttranslational modification, protein turnover, chaperones[V]; Defense mechanism and Signal transduction mechanisms[T]; in the Metabolism, the up-regulated genes involved in Energy production and conversion[C]; Carbohydrate transport and metabolism[G] and Inorganic ion transport and metabolism[P]. Meantime, in the Information storage and processing, the down-regulated genes involved in Translation, ribosomal structure and biogenesis[J] and Replication, recombination and repair [L]; in the Cellular processes and signaling, the down-regulated genes involved in Posttranslational modification, protein turnover, chaperones[0]; in the Metabolism, the down-regulated genes involved in Carbohydrate transport and metabolism[G]; Energy production and conversion[C]; Amino acid transport and metabolism[E]. Compared with the up-regulated gene, the pathway of the down-regulated gene was significantly less than that of the up-regulated gene, only enriched to 4 metabolic pathways, ribosome, Flavonoid biosynthesis, Carbon fixation in photosynthetic organisms and one carbon pool by folate (Fig $3 \mathrm{~b}$ ). The up-regulated genes are enriched in Photosynthesis in the KEGG database, Phenylpropanoid biosynthesis, Plant hormone signal transduction, Starch and sucrose metabolism, Nitrogen metabolism, ABC transporters, Oxidative phosphorylation, Nicotinate and nicotinamide metabolism and Cyanoamino acid metabolism. 6,985 unigenes were annotated in the KEGG database and were assigned to 14 pathways. We studied explicitly the complex biological factors of genes and obtained pathway annotations for unigenes. (Fig 3c). In summary, the upregulated genes were mainly involved in Cell wall/membrane biogenesis and Plant hormone signal transduction after UV treatment, which might promote plant metabolism and accelerates plant growth. 
According to the results of KOG and KEGG, DEGs related to the Photosynthesis pathway, Plant auxin and Phenylpropanoid biosynthesis pathways (associated with cell wall biogenesis) were analyzed. In the field of plant hormone signal transduction, the related genes from the auxin response protein aux/iaa, and Saur family protein were upregulated (Fig 4), which could promote the elongation and growth of plant cells and accelerate plant growth. The CRE1 (EC: 2.7.13.3) high expression is involved in zeatin biosynthesis metabolism, which could also promote cell division and then accelerates plant growth.

In phenylpropanoid biosynthesis pathway, peroxidase [EC:1.11.1.7], beta-glucosidase [EC:3.2.1.21], and caffeic acid 3-0-methyltransferase [EC:2.1.1.68] were upregulated, and involved mostly in the biosynthesis of guaiacyl- lignin and 5-hydroxy-guaiacyl lignin (Fig 5).

\section{Validation and expression analyses of key enzyme genes}

To verify the changes of gene expression analyses, 10 candidate unigenes associated with leaf development were randomly selected, including the photosynthesis pathway (unigene TR10048 and unigene TR19418), plant auxin signal pathway (unigene TR11070 and unigene TR14945), phenylpropanoid biosynthesis (unigene TR11546, unigene TR11244, unigene TR11203, and unigene TR10148), and flavonoid biosynthetic process (unigene TR8189 and unigene TR15898). As shown in Fig. 6 , these results of genes expression by RT-qPCR were identical to the differential expression from two libraries exhibited. Thus, it is useful to further investigate the genes related to leaf growth in Eucommia ulmoides Oliver (EUO).

\section{Discussion}

In functional traits, plants often exhibit considerable variations in order to match the change environments and maintain normal physiological activities [Franks and Beerling 2009; Zhang et al 2012]. Leaf development trait serves as a model for plant developmental flexibility [Reich 2014]. Studies of leaf traits could greatly improve our understanding of leaf function and plant performance [Blackman et al.2016; Chai et al 2015]. In our study, the change of leaf area and leaf thickness was increased (Table 2), this result should be closely related to the anatomical observation of leaves. The palisade tissue became long and loose, and the spaces between cells increased (Fig 1) in the experimental condition. Schmalstig and McAuslane [2001] suggested because of the appearance of the space between the upper epidermis and palisade tissue, the leaf area and leaf thickness increased, the additional air space could alter light reflection promote the utilization of sunlight for plant growth. Moreover, the increase in leaf area and leaf thickness could result in utilizing additional $\mathrm{CO}_{2}$ to increase the rate of photosynthesis, leading to better development of leaves, and then promote the increase of leaf dry weight and fresh weight (Table 2). Therefore, it was beneficial to the growth and development of leaves after UV-B radiation.

Plant morphogenic responses are induced after UV-B radiation, and appear to be adaptive, such as the rapid induction of a variety of genes expression, including those involved in plant hormone metabolism and phenylpropanoid synthesis [Hectors et al.2007; Wargent et al.2009; Zhu et al.2016]. Our KEGG 
pathway analysis showed that the differentially up -regulation expressed genes focused on these two aspects. Ido Shwartz et al [Ido et al.2016] specifically emphasized the balance of plant hormones between morphogenesis and differentiation during leaf development, Auxin plays a role in nearly all plant developmental processes, and leaf development is no exception. Thus, we speculate that the increase of the genes expression in auxin signaling pathway (Fig 4) could accelerate leaf growth and increase leaf area in our study (Table 2). Furthermore, lignins primarily provide rigidity and strength to the cell wall and are also involved in defense mechanisms against biotic and abiotic stresses [Neutelings 2011]. Lignin biosynthesis is a complex network encompassing the phenylpropanoid pathway [Hisano et al.2009], the activity of peroxidase (POD) in lignin biosynthesis catalyzes the polymerization of lignin monomer, as the last step of lignin synthesis [Bonawitz and Chapple 2010; Zhao and Dixon 2011]. In our research, the upregulation gene encoding peroxidase [EC:1.11.1.7] (Fig 5) could improve lignins metabolic biosynthesis, which might change the structure of plant cell walls and increase leaf thickness(Table 2), enhance the resistance abilities of plants after UV-B treatment. It is helpful to provide a good method for popularizing the planting the ULMEP in northeast China.

\section{Conclusions}

To identify the genes related to leaf growth traits and biomass, leaf transcriptome for Eucommia ulmoides Oliver (EUO) were studied and analyzed by Illumina sequencing technology. In this study, leaf morphology analysis results showed that leaf area and thickness significant increase $(p<0.05)$ after UV-B radiation; Anatomical results showed that cells from the leaf epidermal and palisade tissue after UV-Btreated plants were also larger than those of control. Two transcriptome databases were integrated, the differentially expressed genes (DEGs) from plant auxin and phenylpropanoid biosynthesis signal pathways were focused on after UV-B treatment. The expression results of RT-qPCR from 10 candidate unigenes were similar to transcriptome database analysis. These results could demonstrate that UV-B radiation is very important to plant using leaf model of Eucommia planting (ULMEP) in Northeast China. Furthermore, this is conducive to increasing the medicinal raw materials and protect tree species from damage.

\section{Declarations}

*Ethics approval and consent to participate Not applicable

\section{*Consent for publication}

all authors have read and agreed to the published version of the manuscript.

\section{*Availability of data and material}

All date generated during the study are interpreted in the manuscript.

\section{*Competing interests}


The authors declare that they have no competing interest.

*Funding The work was supported by Heilongjiang province natural science fund project (No. QC2017009); the Foundamental Research Funds for the Central Universities (No. 2572015CA03) and the Special Fund for Forest Scientific Research in the Public Welfare (201504701-2).

*Authors' contributions Conceptualization, Zhonghua Tang (Z.H.T) and Yusen Zhao (Y.S.Z) ; methodology, Ying Liu (Y. L.) and Dewen Li (D.W L.) ; software, Ying Liu (Y. L.); validation, Yuangang Zu (Y.G.Z). and Yusen Zhao(Y.S.Z); formal analysis, Dandan Du(D.D.D); investigation, Dewen Li (D.W L.); resources, Lili Yu(L.L.Y); data curation, Dandan Du(D.D.D); writing-original draft preparation, Ying Liu (Y. L.); writing-review and editing, Dewen Li (D.W L.); visualization, Yuangang Zu (Y.G.Z); supervision, Yusen Zhao(Y.S.Z); project administration, X.X.Zhonghua Tang (Z.H.T); funding acquisition, Dewen Li (D.W L.). All authors have seen the manuscript and approved to submit to your journal.

* Authors' information (optional) Denwen Li (Associate professor) and Ying Liu (Ph D), Key Laboratory of Forest Plant Ecology, Ministry of Education, Northeast Forestry University, No. 26 Hexing Road, DongLi District, Harbin 150040, China.

E-mail: Idw8182@163.com (D.L.);

arrive100@163.com (Y.L.);

Dudan12@163.com (D.D.);

yugang-zu@163.com (Y.Z.);

tangnefu@163.com (Z.T.);

zhaoysnefu@126.com (Y.Z.)

\section{*Acknowledgements}

Not applicable

\section{References}

Wang L, HY Du, TZ Li, TN Wuyun (2018). De novo transcriptome sequencing and identification of genes related to salt stress in Eucommia ulmoides Oliver. Trees. 32:151-163.

Zhu MQ, WZ Xua, JL Wen, YH Zhu, Y Li, YQ Su, Q Zhang, RC Sun (2017). Dynamic changes of photosynthetic properties and chemicalcompositions of Eucommia ulmoides Oliver under two planting models. Industrial Crops and Products. 96: 46-56.

Dotto M, P Casati (2017). Developmental reprogramming by UV-B radiation in plants. Plant Science. 264: 96-101. 
Rinella MJ, JJ James (2017). A modelling framework for improving plant establishment during ecological restoration. Ecological Modelling. 361: 177-183.

Hectors K, E Prinsen, W De Coen, MAK Jansen, Y Guisez (2007). Arabidopsis thaliana plants acclimated to low dose rates of ultraviolet $B$ radiation show specific changes in morphology and gene expression in the absence of stress symptoms. New Phytol.175: 255-270.

Wargent JJ, VC Gegas, GI Jenkins, JH Doonan, ND Paul (2009). UVR8 in Arabidopsis thaliana regulates multiple aspects of cellular differentiation during leaf development in response to ultraviolet $\mathrm{B}$ radiation. New Phytol.183:315-326.

Zhu MQ, JL Wen, YH Zhu, YQ Su, RC Sun (2016). Isolation and analysis of four constituents from barks and leaves of Eucommia ulmoides Oliver by amulti-step process. Ind. Crops Prod. 83:124-132.

Grabherr MG, Haas BJ, Yassour M, Levin JZ, Thompson DA, Amit I (2011). Full length transcriptome assembly from RNA-seq data without a reference genome. Nat. Biotechnol. 29: 644-652.

Liu, H.M.; Fu, J.M.; Du, H.Y.; Hu, J.J.; Wu, T.Y(2016) De novo sequencing of Eucommia ulmoides flower bud transcriptomes for identification of genes related to floral development. Genomics Data. 9:105-110.

Conesa A, Götz S, García-Gómez JM, Terol J, Talón M, Robles M (2005) Blast2 G0: a universal tool for annotation, visualization and analysis in functional genomics research. Bioinformatics. 21:3674-3676.

Ye J, Fang L, Zheng H, Zhang Y, Chen J, Zhang Z (2006) WEGO: a web tool for plotting GO annotations. Nucleic Acids Res. 34: 293-297.

Zhang JX, He HM, Wu KL,Silva TD, Zeng SJ, Zhang XH, Yu ZM, Xia HQ, Duan J (2016)Transcriptome analysis of Dendrobium officinale and its application to the identification of genes associated with polysaccharide synthesis.Frontier in plant science.7:1-15.

Franks PJ, Beerling DJ (2009) Maximum leaf conductance driven by CO2 effects on stomatal size and density over geologic time. Proc. Natl. Acad. Sci. 106: 10343-10347.

Zhang SB, Guan ZJ, Sun M, Zhang JJ, Cao KF, Hu H (2012) Evolutionary association of stomatal traits with leaf vein density in Paphiopedilum, Orchidaceae. PLoS One. 7(6): e40080.

Reich PB (2014). The world-wide 'fas-slow' plant economics spectrum: a traits manifesto. J. Ecol. 102: 275-301.

Blackman CJ, Aspinwall MJ, Resco de Dios V,Smith RA,Tissue DT.(2016) Leaf photosynthetic, economics and hydraulic traits are decoupled among genotypes of a widespread species of eucalypt grown under ambient and elevated CO2. Funct. Ecol. 30: 1491-1500. 
Chai Y, X Zhang, Yue M, Liu X, Li Q, Shang H, Meng Q, Zhang R.(2015) Leaf traits suggest different ecological strategies for two Quercus species along an altitudinal gradient in the Qinling Mountains. J. For. Res. 20: 501-513.

Schmalstig JG, HJ McAuslane (2001). Developmental anatomy of zucchini leaves with squash silver leaf disorder caused by the silver leaf white fly. J. Am. Soc. Hortic. Sci. 126:544-554.

Ido S, M Levy, O Naomi, B Maya (2016). Hormones in tomato leaf development. Developmental Biology. 419: $132-142$.

Neutelings G (2011). Lignin variability in plant cell wall: contribution of new model. Plant Sci. 181:379386.

Hisano N, R Nandakumar, Z Wang (2009). Genetic modification of lignin biosynthesis for improved biofuel production. In vitro cellular and developmental biology. 45:306-313.

Bonawitz ND, C Chapple (2010). The Genetics of Lignin Biosynthesis: Connecting genotype to phenotype. Annu rev Genet. 44:337-363.

Zhao Qc, RA Dixon (2011). Transcriptional networks for lignin biosynthesis: more complex than we thought? Trends in Plant Science.16:227-233.

\section{Figures}



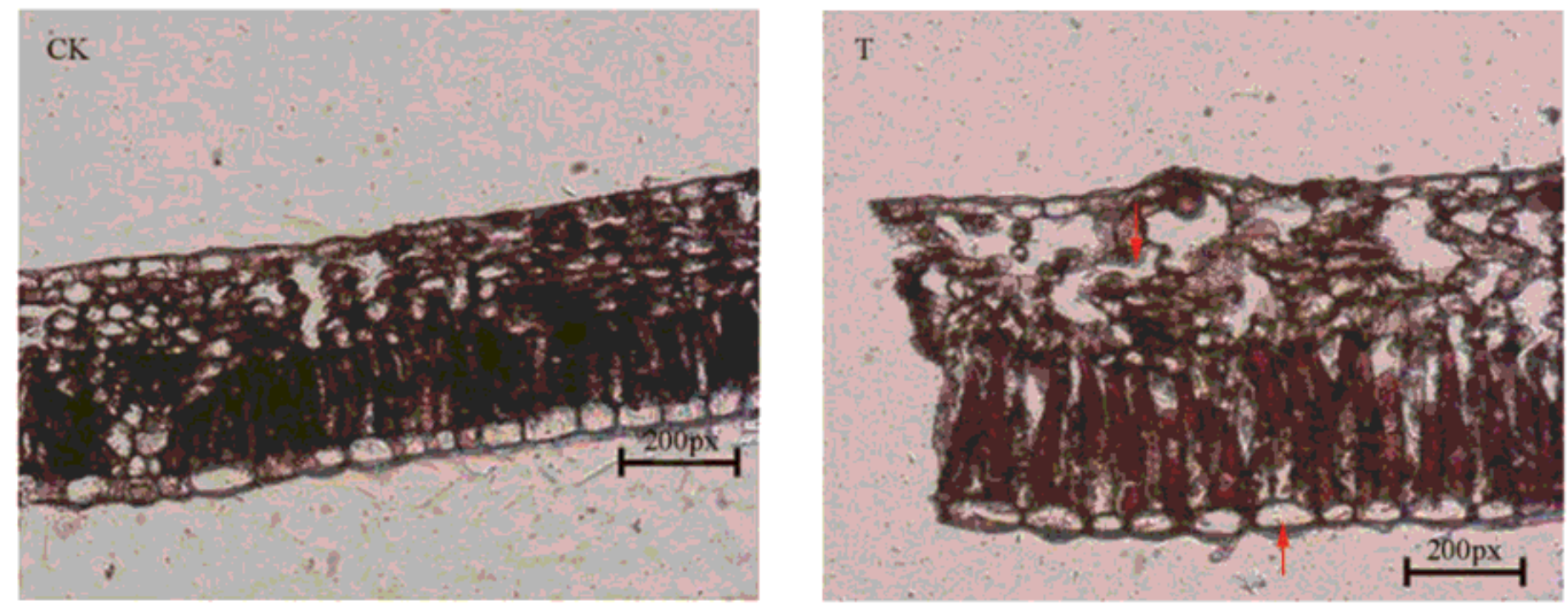

\begin{tabular}{ccc}
\hline \multirow{2}{*}{ Samples } & \multicolumn{2}{c}{ Cross cutting } \\
& epidermal cell (px) & palisade tissue (px) \\
\hline $\mathrm{CK}$ & $80 \pm 4 \mathrm{~b}$ & $145.45 \pm 7.27 \mathrm{~b}$ \\
$\mathrm{~T}$ & $166.67 \pm 8.33 \mathrm{a}$ & $364.64 \pm 18.23 \mathrm{a}$ \\
\hline
\end{tabular}

Note:Effects on the size of epidermal cells and palisade tissue after UV-B radiation treatment, different lower-case letters indicate significant differences according to Duncan's multiple test at $\mathrm{P}<0.05$.

Figure 1

Anatomical characteristics of EUO leaves and the size of epidermal cells and palisade tissue CK, Anatomical characteristics of leaves under natural light (bar= 200 px); T, anatomical characteristics of leaves after UV-B treatment (bar= 200 px). 


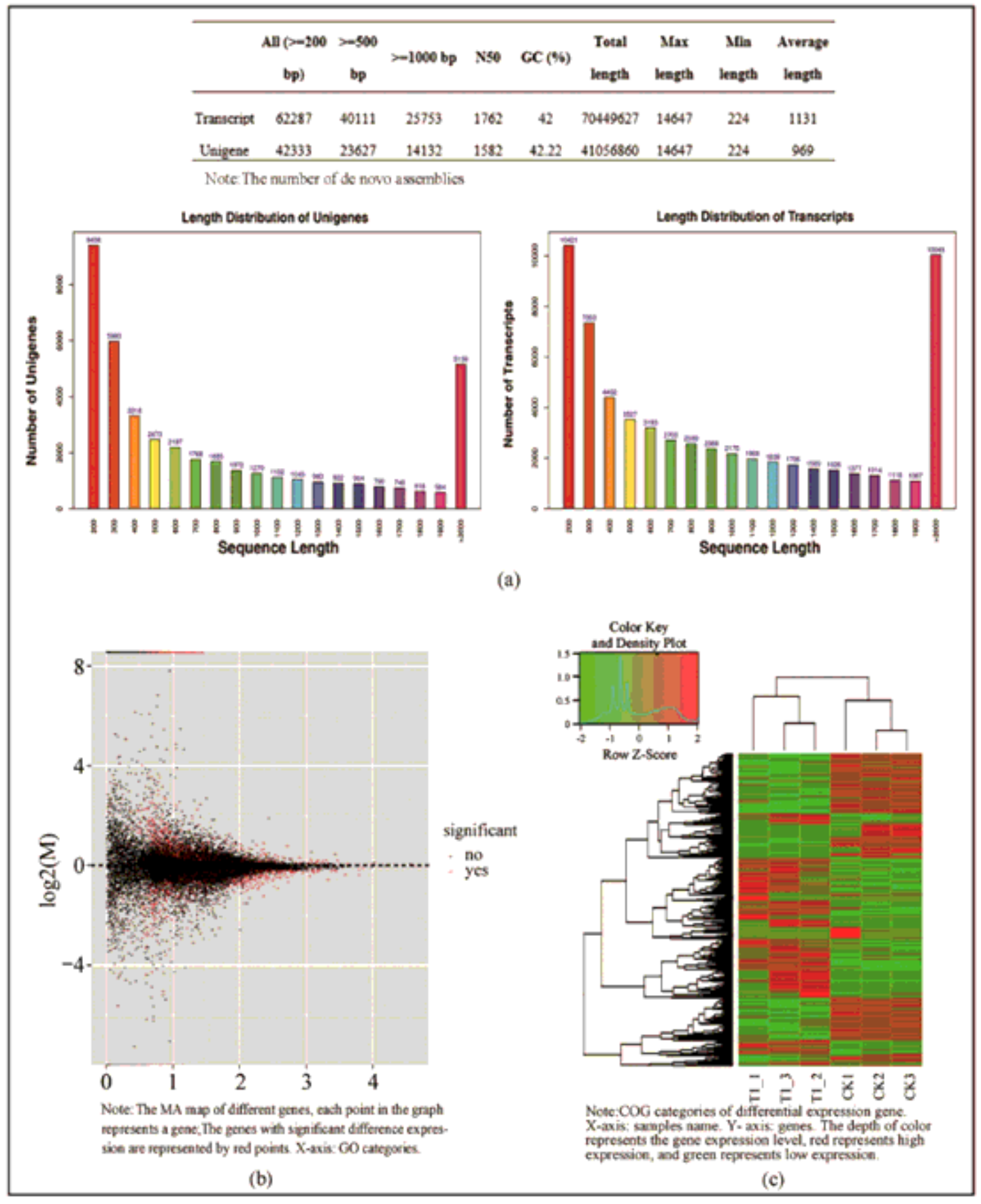

Figure 2

Length distributions of all assembled transcripts and unigenes (a), MA map (b) and COG categories of differential expression gene (c). 


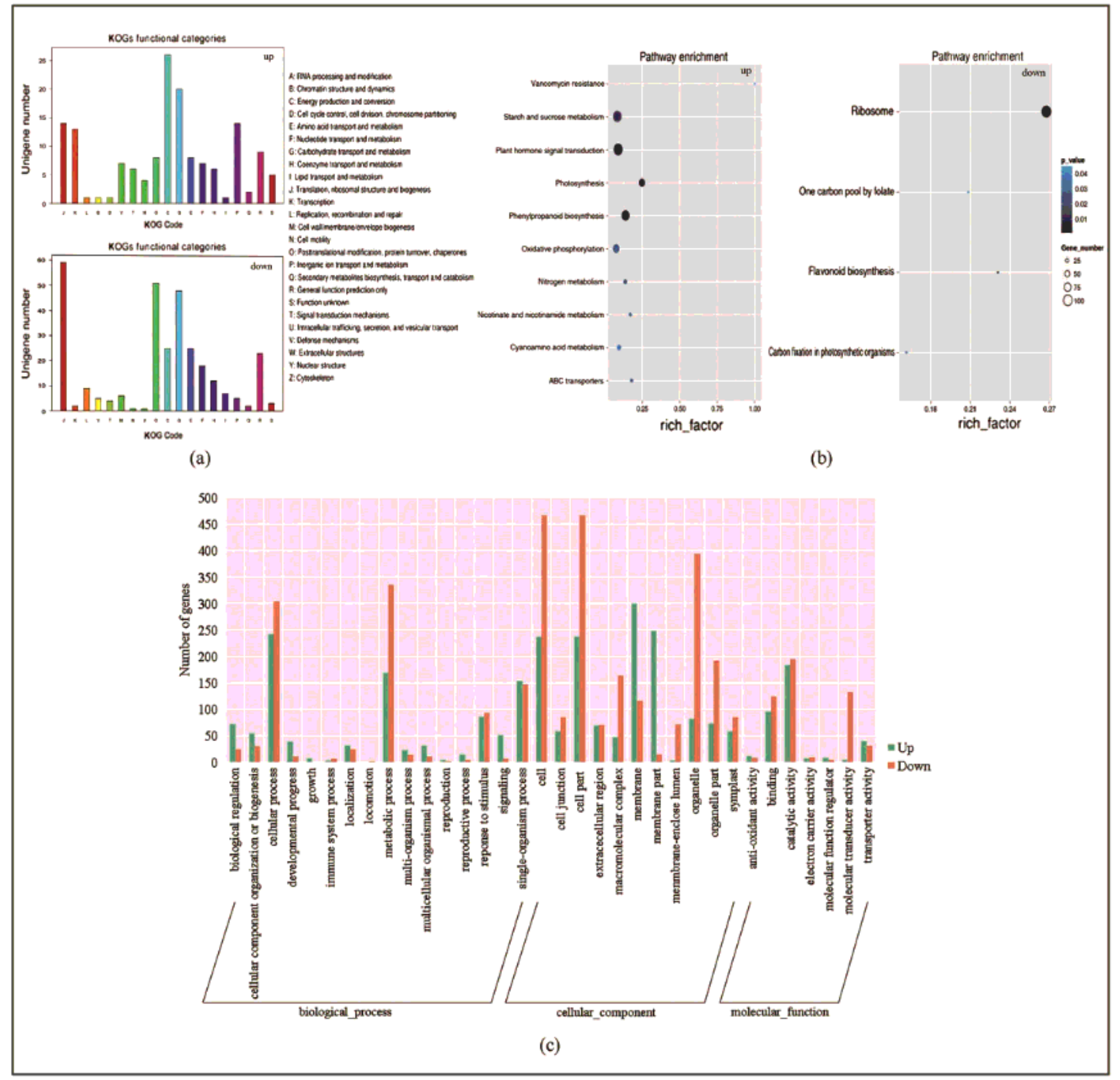

Figure 3

Number distribution and pathway analysis of differentially expressed genes. 
PLANT HORMONE SIGNAL TRANSDUCTION

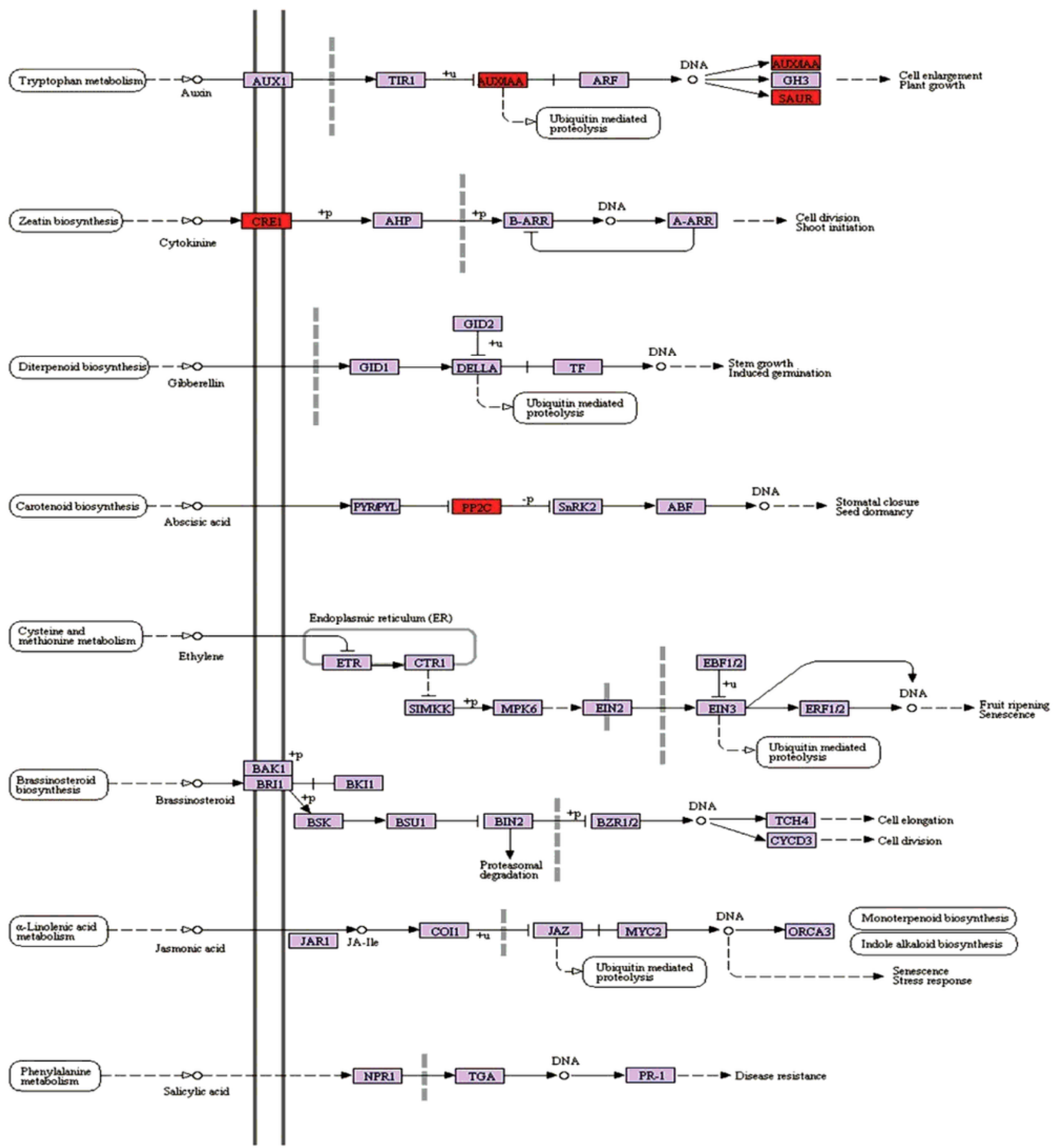

0407599616 16
(c) Kanehesisa Lsboratories

\section{Figure 4}

Network analysis of various auxins involved in the regulation of the leaf development process. the red color indicates the key upregulated genes associated with the auxin signaling pathway. 


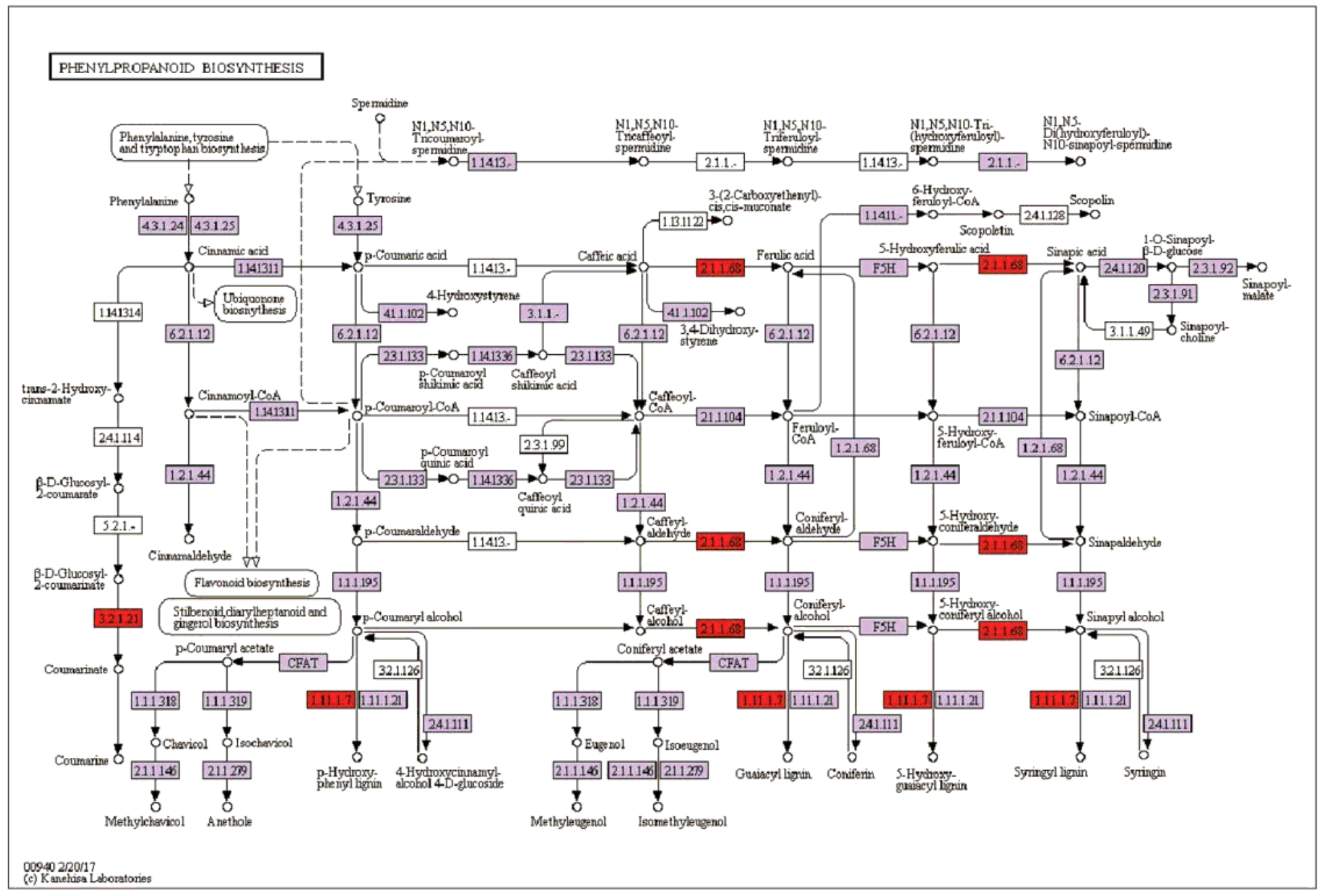

\section{Figure 5}

The putative phenylpropanoid biosynthesis metabolic pathway of EUO was constructed based on KEGG annotation. These unigenes are distributed in the rectangular boxes in the figure. The red color indicates the key upregulated genes associated with the lignin metabolic pathway. 


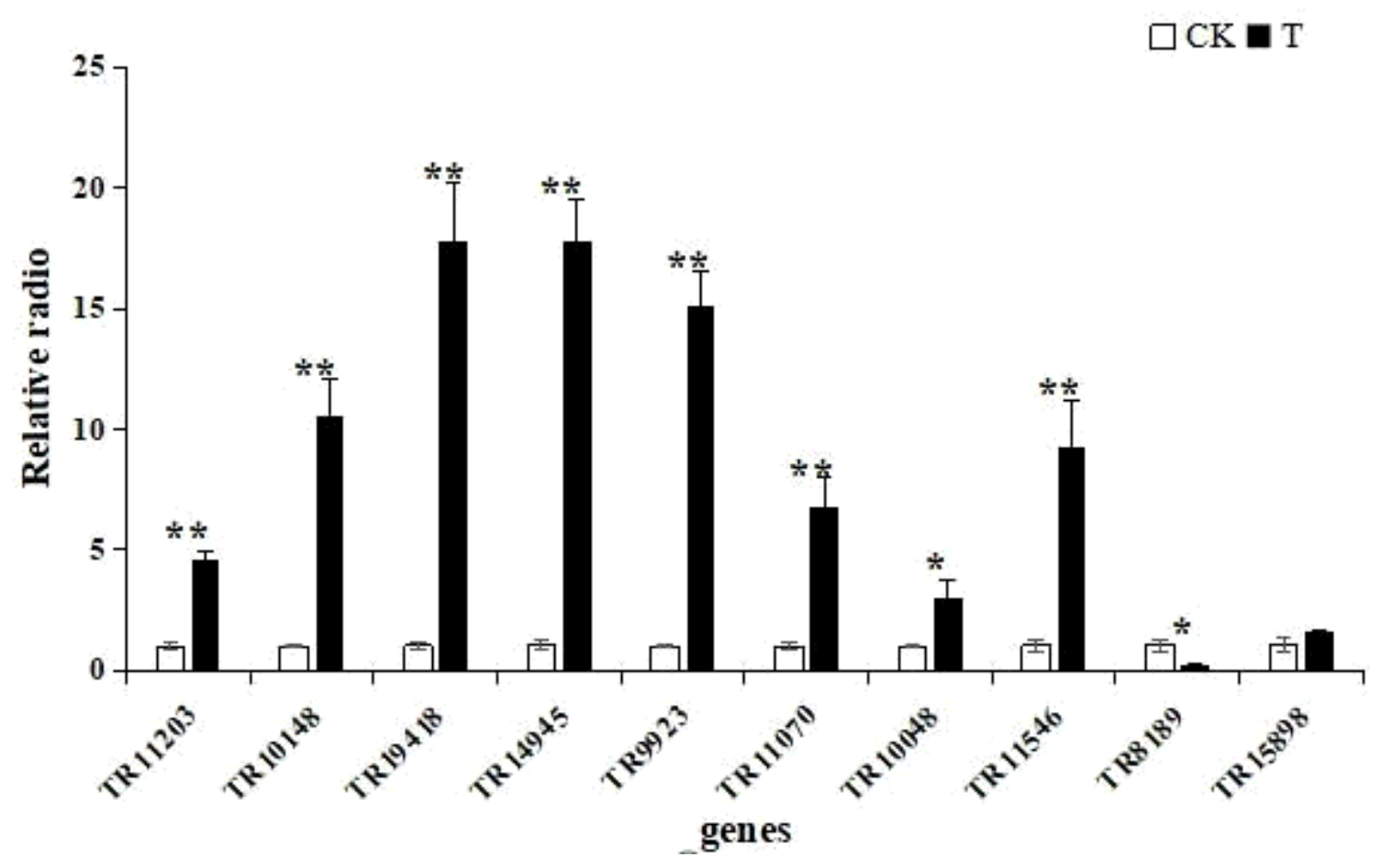

Figure 6

The 10 candidate unigenes associated with leaf development were detected under the two treatments. (Ribosomal 40S protein S9 as an internal control and CK as the control). 